\title{
BroilerBreak!: Partitioning ME in Broiler Breeders
}

\author{
Airell G. C. DesLauriers, Martin J. Zuidhof, Valerie Carney, and Brenda L. Schneider \\ Department of Agricultural, Food \& Nutritional Science, University of Alberta
}

\begin{abstract}
Feed restriction is a common tool utilized in broiler breeder production. The period of most severe feed restriction is during the rearing phase. In order to feed at the proper requirements the maintenance requirements of the birds must be estimated. Biometric models have been previously developed, however these estimates may loose accuracy due to changes in genetic selection criteria. In order to verify the estimates of metabolizable energy of maintenance (MEm), a study was conducted from 0-20 weeks with a total of 8,500 Ross 308 females placed at two separate commercial farms. The study also examined the effect of temperature on maintenance requirements of commercial broiler breeders. Although the effect of temperature was inconclusive, the estimates of maintenance requirements were consistent with other experimental findings in the literature.
\end{abstract}

\section{INTRODUCTION}

In the poultry production chickens are utilized for 2 main products, meat and eggs. Birds that are used for eggs for consumption are called leghorns. These birds have been selected for a high egg production of the large size with completely calcified shells. Broilers are birds that are raised for meat production and have a large proportion of their resources dedicated to breast meat production. Broilers are the offspring from broiler breeders, which have the same genetic growth potential as broilers. A major concern with the management of broiler breeders is the control of body weight during the rearing and reproduction phases. Broiler breeders are feed restricted due to the negative relationship between adipose tissue and egg production [1,2]. Excess energy intake is deposited largely as fat therefore to prevent excessive fat deposition broiler breeders are fed close to maintenance requirements. Broiler breeders are most intensively feed restricted during the rearing phase of development. This stage is critical in programming the egg production, health status, and longevity of the bird, making it vital to correctly determine the degree of feed restriction during this period.

As broiler breeder feeding levels are only slightly above maintenance requirements, changes in the weather can result in over- or under-feeding of broiler breeders. Since broiler breeders are feed restricted to their minimum required level to prevent excess growth, it is important to understand how common production decisions impact later growth and yield of broiler breeders. Mathematical models can be used to demonstrate the effect a production decision has on subsequent growth.

Bioeconomic models have been developed with production parameters however there is growing interest for the development of biometrical models. Biometrical models are mathematical formulas that predict biological features of a flock or individual bird [3]. There is interest in biometrical models because economic models are dependent on biometrical models. Changes in genetics have caused older biometric models to become out-dated. Biometrical models have been developed for broiler breeders [4], and broilers [5]. A broiler breeder model has been developed through raising the animals in an intensively monitored research facility [4]. In order for biometric models to be accurate enough to use in the industry, the mathematical model must be validated. One of the purposes of our study was to validate coefficients of metabolizable energy of maintenance $\left(\mathrm{ME}_{m}\right)$ obtained in previous studies. Validating the model with industry data will allow for the model to be tested against other flocks.

Historically, $\mathrm{ME}_{m}$ is expressed as a function of metabolic bodyweight $\left(\mathrm{BW}^{0.75}\right)$. However, recent studies have determined that $\mathrm{ME}_{m}$ is more accurately represented by exponents other than 0.75 in broilers [6]. Therefore, in our study, $\mathrm{ME}_{m}$ will be reported as a function of $\mathrm{BW}^{0.67}$.

\section{MATERIALS AND METHODS}

The data were obtained through the use of datasets obtained from industry flocks. Two farms data were included in this analysis. For confidentiality, they are referred to as Farm A and Farm B. In Farm A 4,500 Ross 308 female broiler breeders were reared in 6800 square feet of floor space (stocking density of $1.51 \mathrm{ft}^{2} / \mathrm{bird}$ ). In Farm B 4,000 Ross 308 female broiler breeders were reared separately in 7930 square feet of floor space for the first 10 weeks (stocking density of $1.98 \mathrm{ft}^{2} / \mathrm{bird}$ ). After 10 weeks, 460 Farm B males were then cohabited with the Farm B females. Project protocol was approved by the University of Albertas Animal Care and Use Committee Livestock division prior to data collection.

Barn dimensions and configurations were recorded prior to chick placement. Each flock was housed in conventional poultry houses (Figure 1,2) and was raised according to industry standards. Body weight (BW) of the flock was estimated through random selection of target birds weighed at placement, 3-4 weeks of age, and 9-11 weeks of age. Additional weekly weighing performed by the producer was also included in the analysis. Temperatures were recorded on a 30min interval, 


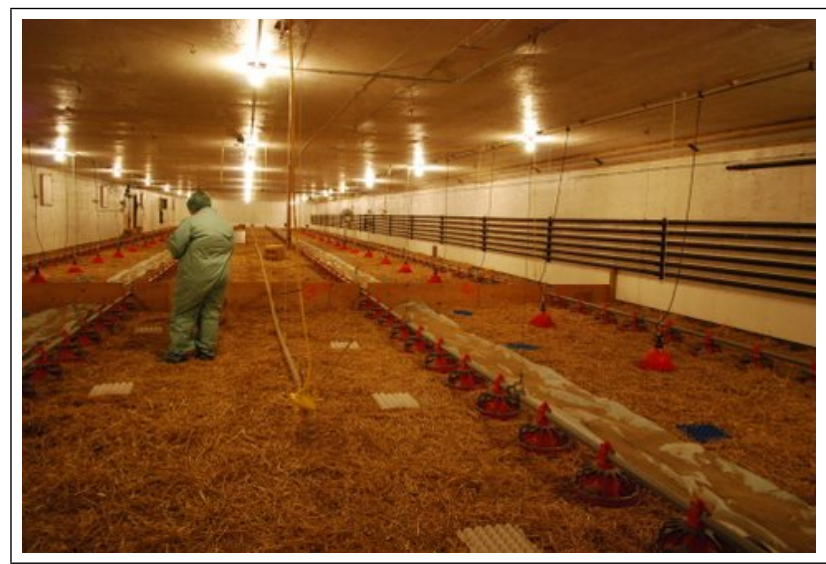

FIG. 1. In-Barn Configuration of Farm A [7]

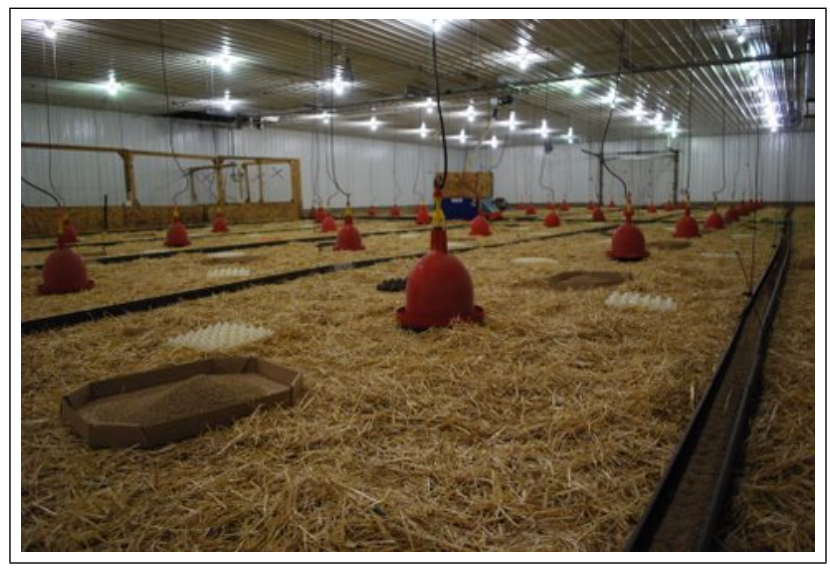

FIG. 2. In-Barn Configuration of Farm B [7]

using automated data loggers positioned at bird head height. Historical external temperature data were obtained from Environment Canada. Using the physical location of the farms, the closest possible weather station was utilized (Three Hills and Linden). The weather stations for Farm A and Farm B were $7.83 \mathrm{~km}$ and 11.67 $\mathrm{km}$ away respectively. A regression was performed to examine the correlation between barn temperature and external temperature.

Data were analysed in a least squares linear model using the mixed procedure of SAS to estimate the metabolizable energy (ME) requirement for maintenance for age in 4 week blocks: 0-4 weeks, 4-8 weeks, 8-12 weeks, 12-16 weeks, and 16-20 weeks to the following linear model.

$$
m e i=A\left(W^{0.67}\right)+b\left(W^{0.67}\right)\left(T_{e}-21\right)+c(G) \quad[4]
$$

Where mei is ME intake ( $\mathrm{kcal} /$ day); $\mathbf{W}$ is $\mathrm{BW}(\mathrm{kg})$; $\mathbf{T}_{e}-21$ is internal barn temperature corrected to 21C; and $\mathrm{G}$ is gain ( $\mathrm{g} /$ day); $\mathbf{A}$ is the coefficient representing the relationship between age specific base ME mainte- nance requirements; $\mathbf{b}$ is the coefficient of the effect of temperature on the maintenance ME energy; $\mathbf{c}$ is the coefficient representing the $\mathrm{ME}$ cost for one gram of $\mathrm{BW}$ gain [4].

\section{RESULTS}

Our results from Farm A show a decrease in the base $\mathrm{ME}_{m}$ requirements from weeks 4-12 and then an increase in $\mathrm{ME}_{m}$ in the period 16-20 weeks (Table 1).

Farm B shows a decrease between the 4-8 week period and the 8-12 week period and then an increase in $\mathrm{ME}_{i} \mathrm{sub}_{i} \mathrm{~m}_{\mathrm{i}}$ / sub $\mathrm{s}_{i}$ throughout the $12-20$ week period (Table 2). For both farms, the highest base $\mathrm{ME}_{m}$ requirements were during the 4-8 week period and the 16-20 week period (Table 1, 2).

The coefficient for $\mathbf{b}$, the effect of temperature on maintenance, was 4.8348 and 4.0291 for Farm A and Farm B respectively. However the effect of temperature on maintenance requirements did not differ significantly. The coefficient for $\mathbf{c}$, the ME requirement per gram of growth, was 0.7007 and 1.3272 respectively. However, this did not differ significantly.

The correlation between external environmental temperature and internal barn temperature was strongest when outdoor temperatures were higher than the set point of the barn. In the first few weeks, when the barn temperature was the most elevated, high external temperature was not significantly correlated to internal barn temperature (Figure 3, 4).

Excluding the first 2 weeks of rearing, the relationship between outdoor daily maximum temperature and indoor daily average temperature was found to be highly correlated for each farm (Farm A R ${ }^{2}=0.7650$, Farm B R ${ }^{2}=$ 0.7582 , Figure 5, 6).

\begin{tabular}{lrrr}
\hline Weeks & \multicolumn{1}{c}{ A } & \multicolumn{1}{c}{ \pm} & P-Value \\
\hline $0-4$ & 97.6639 & 74.148 & 0.2203 \\
$4-8$ & 128.91 & 44.6868 & 0.018 \\
$8-12$ & 107.13 & 44.6747 & 0.04 \\
$12-16$ & 76.9054 & 37.2027 & 0.0687 \\
$16-20$ & 132.83 & 16.1646 & $<0.0001$ \\
\hline
\end{tabular}

TABLE I. Estimated coefficients values of $\mathbf{A}$ for Farm A; the coefficient representing the relationship between age specific base ME maintenance requirements.

\begin{tabular}{lrrr}
\hline Weeks & \multicolumn{1}{c}{ A } & \multicolumn{1}{c}{ \pm} & P-Value \\
\hline $0-4$ & - & - & - \\
$4-8$ & 88.3218 & 9.8596 & $<.0001$ \\
$8-12$ & 55.7727 & 11.6257 & 0.002 \\
$12-16$ & 63.5079 & 8.3268 & 0.0001 \\
$16-20$ & 72.9209 & 9.1673 & $<.0001$ \\
\hline
\end{tabular}

TABLE II. Estimated coefficients values of A for Farm B; the coefficient representing the relationship between age specific base ME maintenance requirements. 


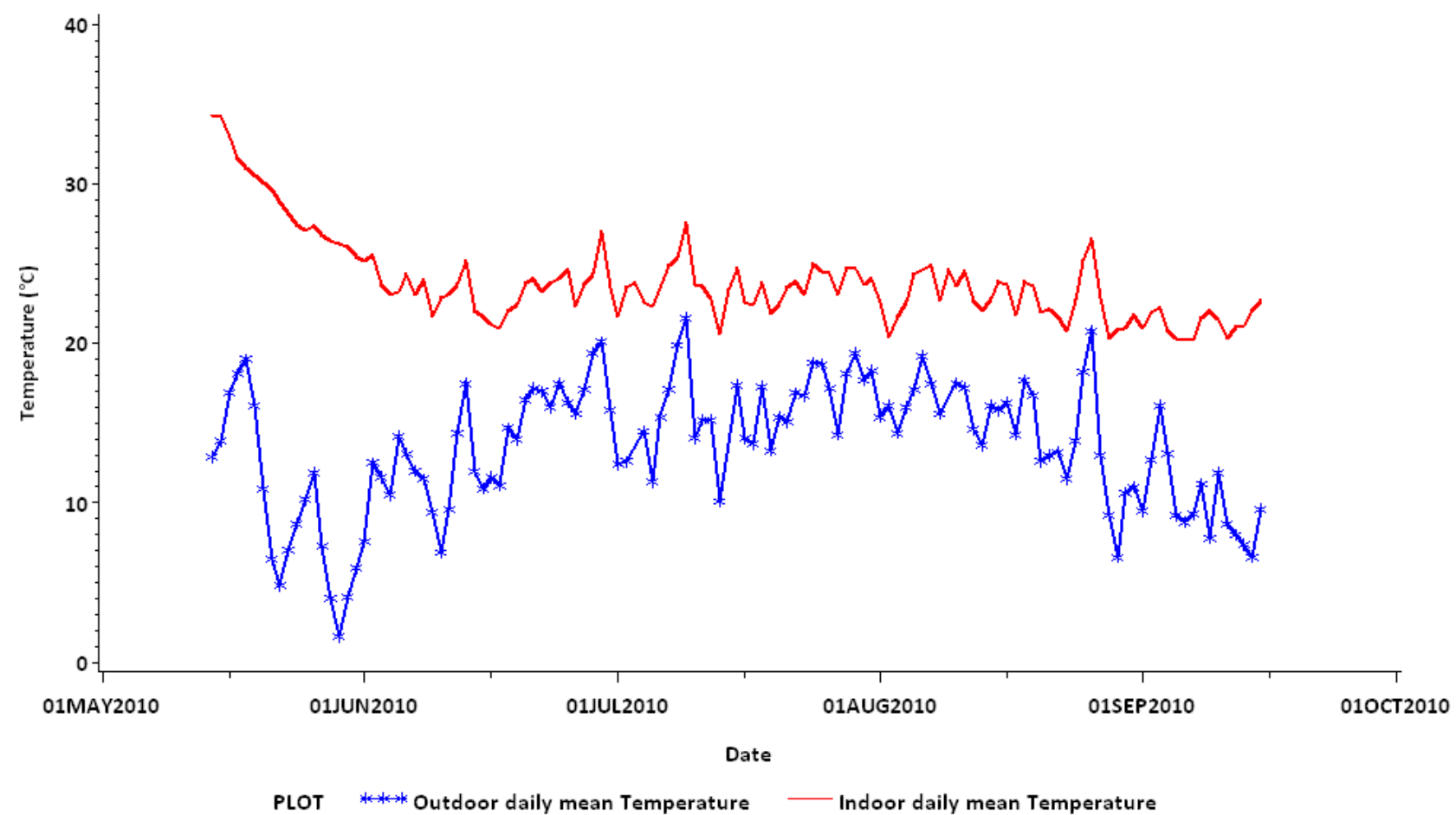

FIG. 3. Relationship between indoor and outdoor barn temperature at Farm A.

\section{DISCUSSION}

The reduction in required $\mathrm{ME}_{m}$ in the period of 8-16 weeks is likely due to the increase in the severity of feed restriction during this period. Broiler breeder flocks are fed ad libitum for the first 2-5 weeks which coincides with the decreased feed efficiency observed during this time. Feed efficiency increases during periods of feed restriction compared to ad libitum feeding [8]. The highest degree of feed restriction is during the 10-14 weeks of age [4]. Studies estimated that at 9-20 weeks of age, $95-96 \%$ of total ME intake was used for maintenance whereas from 5-8 weeks $72.5 \%$ was utilized for maintenance [9]. Feed intake has been found to be positively correlated to $\mathrm{ME}_{m}$ [10]. Through reduced feed intake $\mathrm{ME}_{m}$ is decreased [10]. This theory supports that the reduction in $\mathrm{ME}_{m}$ during the $8-16$ period is due to feed restriction relative to ad libitum.

Decreases in the efficiency of the flock are seen during the 16-20 week period in both Farm A and Farm B (Table $1,2)$. During the 16-20 week period, the degree of feed restriction is reduced causing an increase in the feed intake relative to metabolic $\mathrm{BW}$ and therefore a higher degree of diet induced thermogenesis. The differences between efficiencies can be explained in the changes in basal metabolic rate (BMR) that happen during the rearing period. Studies in rats have shown that the basal metabolic rate of restricted rats was significantly lower than that of ad libitum fed rats [11]. Work by Forsum et al. [11] can be compared to broiler breeders which begin with ad libitum feeding early in life. Broiler Breeders are feed restricted later in life to meet the required growth curve and therefore their basal metabolic rate may decrease, as seen in the Forsum et al. [11] study.

It is likely that, because of the small variation in temperature over the course of the trials, the relationship between temperature and metabolic BW was not significant. Although our study showed inconclusive results for the effect of temperature on maintenance requirements, temperature has been shown to affect $\mathrm{ME}_{m}$ $[4,12,13]$. Due to the severity of feed restriction, which is very close to requirements, any change in $\mathrm{ME}_{m}$ must be accounted for as it will affect energy available for growth. In feed restricted broiler breeders at 16 wks of age, $93 \%$ of metabolizable energy intake is utilized for maintenance [4]. Since broiler breeders are homeothermic, temperature fluctuations from 15 to $17 \mathrm{C}$ may require a $5 \%$ change in feed allocation [4]. Higher outdoor temperatures have more of an influence on indoor barn temperatures except during the first few weeks of rearing where indoor barn temperatures are high (Figure 3,4$)$. In order to adjust for these fluctuations, the weather forecast should be taken into consideration prior to making feeding decisions.

Interestingly, the data obtained from industry sources was similar to other studies of the Ross 708 (Zuidhof nutrition conference) and the Hubbard-Hi-Yield broiler breeder [9]. However, previous research has determined slightly higher coefficients for $\mathrm{ME}_{m}$ than our study. In our study, temperatures were controlled as per broiler 


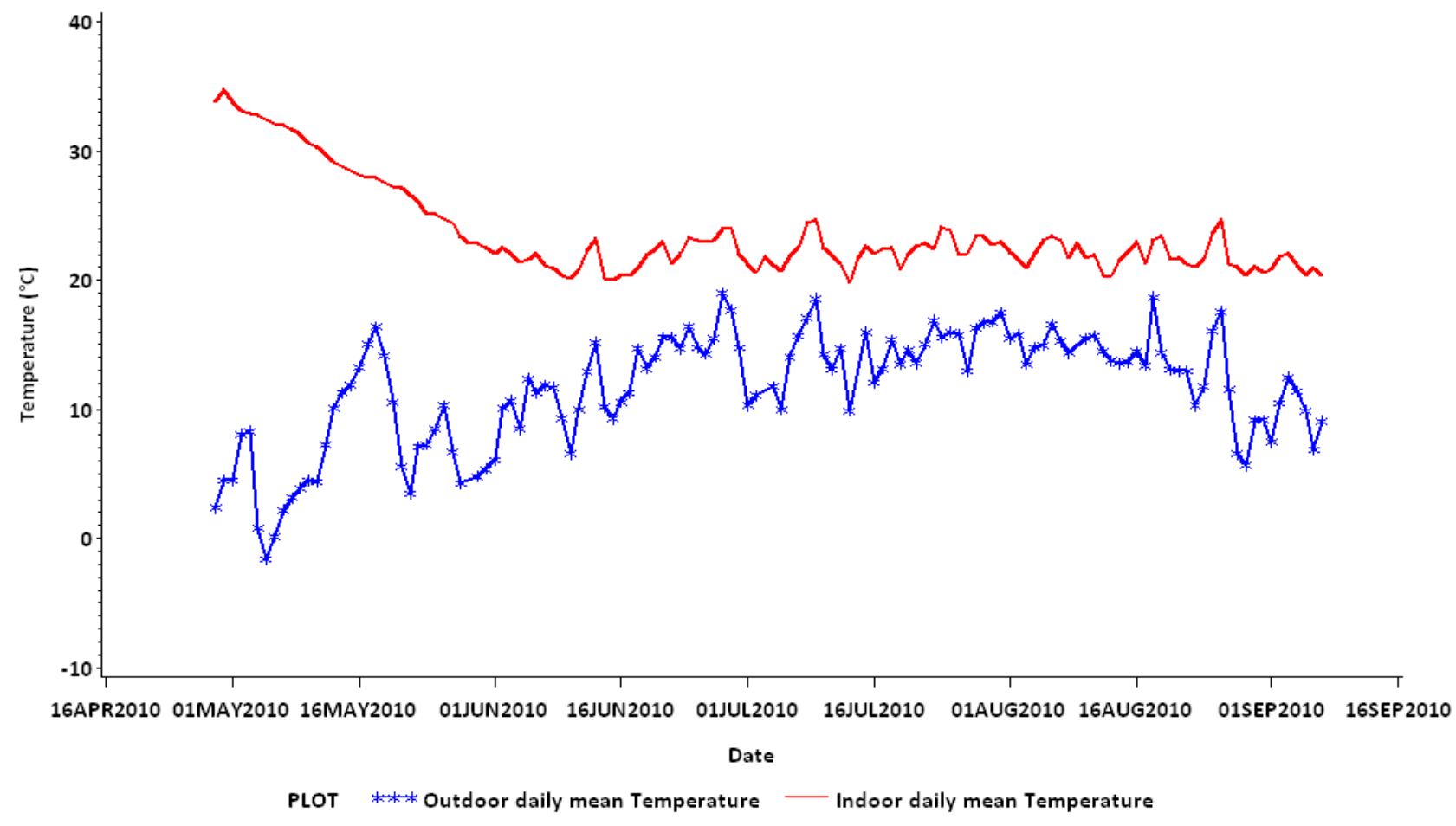

FIG. 4. Relationship between indoor and outdoor barn temperature at Farm B.

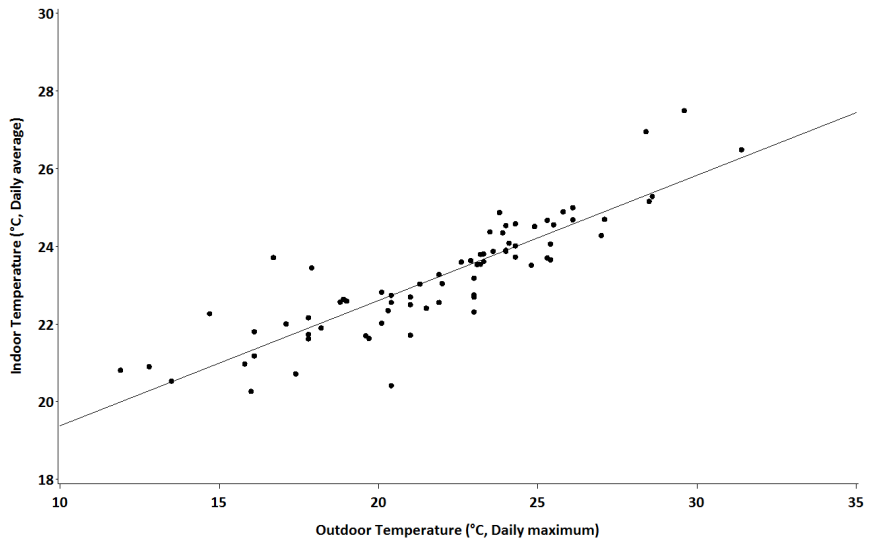

FIG. 5. Correlation between Indoor and Outdoor temperature at Farm $\mathrm{A}\left(\mathrm{R}^{2}=0.7650\right)$.

breeder handbook recommendations and the poultry were not subjected to differing temperatures. The inconsistencies may also be due to the differences in adipose and muscle tissue ratios that are maintained by broiler breeders. Further composition of body tissues could be examined as lipid tissue is less expensive to maintain than muscle tissue.

Another possible inconsistency is the energy density and source of ME in each study. This will leave the efficiency in which different energy sources are utilized as

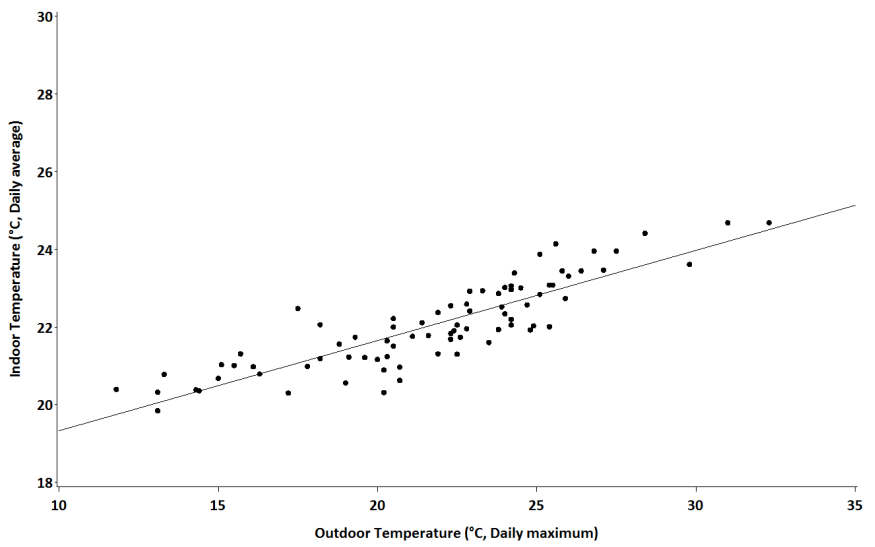

FIG. 6. Correlation between Indoor and Outdoor temperature at Farm $B\left(R^{2}=0.7582\right)$.

a confounding variable [14].

Unlike Zuidhof et al. [4] the sources of error in our study may be resultant from errors in our estimates of flock BW. Variations in sample BW may have been introduced when selecting birds at random in the barn. A possible bias would arise because heavier breeders may move slower making them easier to catch.

It is interesting to note that, after a researcher weighing, the average daily gain of the flock had a significant decrease. However this decrease was not observed 
after the producer weighed the flock. The decrease may have been caused through inconsistency in BW obtained between the producer and the researcher leading to the over estimation of BW by the researcher and subsequently a reduction in feeding by the producer. Due to bodyweight fluctuations the broiler breeders metabolism will be constantly interchanging from a positive energy balance to a negative energy balance impacting the BMR of the flock.

The importance of understanding how production decisions impact later growth in broiler breeders is extremely important. Through the continued development and improvement of mathematical models these decisions can be accurately tailored to increase overall efficiency.

\section{REFERENCES}

[1] Brody, T., Y. Eitan, M. Soller, I. Nir, and Z. Nitsan. 1980. Compensatory growth and sexual maturity in broiler females reared under severe food restriction from day of hatching. Br. Poult. Sci. 21:437-446.

[2] Whitehead, C. 2002. Nutrition and poultry welfare. World's Poult. Sci. J. 58:349-356.

[3] Zoons, J., J. Buyse, and E. Decuypere. 1991. Mathematical models in broiler raising. World's Poult. Sci. J. 47:243-255.

[4] Zuidhof, M.J., A. Pishnamazi, C. Ouellette, D. Paul, and R. A. Renema. 2010. Diet $x$ temperature interactions in broiler breeders and broilers. Pages 128136 in Proc. Of Calif. Anim. Nutr. Conf., Fresno, CA.

[5] Zuidhof, M. J. 2005. Mathematical characterization of broiler carcass yield dynamics. Poult. Sci. 84:1108-
1122.

[6] van Milgen, J., J. Noblet, S. Dubois, B. Carr, and H. Juin. 2001. Utilization of metabolizable energy in broilers. Poult. Sci. 80 (Suppl.1), 170 (Abstr.).

[7] Schneider, B. 2011. Agric. and Rural Dev. Alberta, Edmonton. Personal communication.

[8] Pinchasov, Y., I. Nir, and Z. Nitsan. 1985. Metabolic and anatomical adaption of heavy-bodied chicks to intermediate feeding. 1. Food Intake, growth rate, organ weight, and body composition. Poult. Sci. 64:20982109.

[9] Sakomura, N. K., R. Silva, H. P. Couto, C. Coon, and C. R. Pacheco. 2003. Modeling metabolizable energy utilization in broiler breeder pullets. Poult. Sci. 82:419427.

[10] Pishnamazi, A., R. A. Renema, M. J. Zuidhof, and F. E. Robinson. 2008. Effect of initial full feeding of broiler breeder pullets on carcass development and body weight variation. Poult. Sci. 17:505-514.

[11] Forsum, E., P. Hillman, and M. Nesheim 1981. Effect of energy restriction on total heat production, basal metabolic rate, and specific dynamic action of food in rats. J. Nutr. 111:16911697.

[12] Costa, M. S. 1981. Fundamental principles of broiler breeders nutrition and the design of feeding programs. World's Poult. Sci. J. 37:177-192.

[13] Luiting, P., 1990. Genetic variation of energy partitioning in laying hens: causes of variation in residual feed consumption. World's Poult. Sci. J. 46:133152.

[14] Noblet, J., X. Shi, and S. Dubois. 1993. Metabolic utilization of dietary energy and nutrients for maintenance energy requirements in sows: basis for a net energy system. Br. J. Nutr. 70:407419. 